\title{
AN ITERATIVE METHOD FOR NUMERICAL INTEGRATION OF RATIONAL FUNCTIONS
}

\author{
DANTE MANNA AND VICTOR H. MOLL
}

\begin{abstract}
We describe a new method for numerical integration of rational functions on the real line. Given a rational integrand, we provide a new rational function preserving its integral on the line. The coefficients of the new function are explicit polynomials in the original ones. These transformations depend on the degree of the input and the desired order of the method. Both parameters are arbitrary. The formulas can be precomputed. Iteration yields an approximation of the desired integral, with $m$-th order convergence. Examples illustrating the automatic generation of these formulas and a comparison with standard numerical schemes are also presented.
\end{abstract}

\section{INTRODUCTION}

The problem of numerical integration of a function over the real line is described in Numerical Analysis texts such as [4, 11]. The standard algorithms for the numerical integration of

$$
I:=\int_{-\infty}^{\infty} F(x) d x
$$

start by dealing with the unboundedness of the domain of integration. This is usually resolved in two ways: the first one considers the problem on a finite interval

$$
I_{L}:=\int_{-L}^{L} F(x) d x
$$

followed by a convergence study as $L \rightarrow \infty$. The alternative is to transform the real line to a bounded interval. For example, the map $t=x /(1+x)$ maps $[0, \infty)$ to $[0,1]$ and then

$$
\begin{aligned}
\int_{-\infty}^{\infty} F(x) d x & =\int_{0}^{\infty}(F(x)+F(-x)) d x \\
& =\int_{0}^{1}\left[F\left(\frac{t}{1-t}\right)+F\left(\frac{t}{t-1}\right)\right] \frac{d t}{(1-t)^{2}}
\end{aligned}
$$

The unboundedness of the original interval of integration is now reflected in the (possible) singularity of the new integrand at the boundary $t=1$. Observe that if the original integrand is a rational function, then so is the new one in (1.3).

In this paper we present a new numerical method for the integration of rational functions on $\mathbb{R}$. It is different in spirit to the standard ones: the numerical approximation to the integral is obtained from a recurrence acting on the coefficients of

Date: March 2, 2022.

1991 Mathematics Subject Classification. Primary 33.

Key words and phrases. Integrals, transformations. 
the integrand. In particular, the integrand is never evaluated. We illustrate the comparison with the standard methods; a more systematic study will be presented elsewhere.

The method presented here is based on a transformation of the coefficients of a rational function, that preserves its integral. This is the so-called rational Landen transformation. The original problem discussed by Landen, Gauss, Legendre and others deals with the elliptic integral

$$
G(a, b)=\int_{0}^{\pi / 2} \frac{d \theta}{\sqrt{a^{2} \cos ^{2} \theta+b^{2} \sin ^{2} \theta}} .
$$

Gauss [5] made the numerical observation that the function $G(a, b)$ was invariant under the transformation

$$
(a, b) \mapsto\left(\frac{a+b}{2}, \sqrt{a b}\right) .
$$

The iteration of (1.5) leads to a numerical evaluation of the elliptic integral, or, as it has been explained by J. and P. Borwein in [3], to efficient methods for the numerical evaluation of $\pi$. The rational analogue of this transformation was developed in 2. 10, and here we show how to use it as a numerical method to evaluate rational integrals. The reader will find in [9] a survey of the diverse aspects related to these transformations.

Section 2 discusses the basic structure of the algorithm. Section 3 introduces a family of polynomials that play an important role in the development of the formulas given in Section 4. Finally, Section 5 contains some examples. The first illustrates the steps for a method of order 2 acting on a rational function of degree 6 . The next two examples illustrate the accuracy of the method and its comparison to the trapezoidal rule. A systematic study of the cost involved in this algorithm will be presented elsewhere.

\section{The LANDEn transformation AND ALgORIthm}

We present a general description of an iterative algorithm for the evaluation of

$$
I:=\int_{-\infty}^{\infty} F(x) d x
$$

Here $F$ is a rational function given as

$$
F(x)=\frac{B(x)}{A(x)},
$$

with

$$
A(x)=\sum_{k=0}^{p} a_{k} x^{p-k} \text { and } B(x)=\sum_{k=0}^{p-2} b_{k} x^{p-2-k},
$$

where $a_{p} \neq 0$. The general construction treats the coefficients $a_{i}$ and $b_{j}$ as indeterminates. Naturally, for specific integrands the parameters $a_{i}$ and $b_{j}$ are real numbers and the maps described in this section are defined on parts of $\mathbb{R}^{2 p}$ where the integrals are convergent.

The set

$\mathcal{R}^{2 p}:\left\{\left(a_{0}, a_{1}, \ldots, a_{p}, b_{0}, \ldots, b_{p-2}\right):\right.$ such that $F$ in (2.2) has finite integral $\}$, 
will be used to represent the rational function $F$ in terms of its coefficients. It will be referred as the coefficient space.

In Section 4 we describe the construction, for each integer $m \geq 2$, of a rational function $F_{1, m}$ that satisfies

$$
\int_{-\infty}^{\infty} F_{1, m}(x) d x=\int_{-\infty}^{\infty} F(x) d x .
$$

The function $F_{1, m}(x)$ has the same degree as the original $F$ and the new coefficients are polynomials in the old coefficients $a_{0}, \ldots, a_{p}, b_{0}, \ldots, b_{p-2}$. Naturally, this produces a map on $\mathcal{R}^{2 p}$ that we denote by $\mathfrak{L}_{m, p}: \mathcal{R}^{2 p} \rightarrow \mathcal{R}^{2 p}$, called the rational Landen transformation of order $m$ and degree $p$. We denote by $\mathfrak{L}_{m, p}^{n}$ its $n$-fold composition. Now introduce a new operator by $\phi: \mathcal{R}^{2 p} \rightarrow \mathbb{R}$ as the map which takes a vector of length $2 p$ corresponding to a rational function in $\mathcal{R}$ of degree $p$ and returns the value of the rational function at $x=0$. In terms of the vector of coefficients, this is the ratio of the last entry over its $(p+1)$-th one. The composition $\phi \circ \mathfrak{L}_{m, p}^{n}$ will be denoted by $\phi_{m, p}^{n}$. The motivation behind $\phi$ is the following: if

$$
F(x)=\frac{b_{0}+b_{1} x+\cdots+b_{p-2} x^{p-2}}{a_{0}+a_{1} x+\cdots+a_{p} x^{p}},
$$

then the function obtained by iterating the map $\mathfrak{L}_{m, p} n$ times is written as

$$
F_{n, m}(x)=\frac{b_{0, n}+b_{1, n} x+\cdots+b_{p-2, n} x^{p-2}}{a_{0, n}+a_{1, n} x+\cdots+a_{p, n} x^{p}} .
$$

The value of $F_{n, m}$ at $x=0$ gives a sequence of real numbers that converges to $1 / \pi$ times the integral of $F$ in (2.5); see 10 for details. The update on this sequence comes from the coefficients of $F_{n, m}$. These are obtained by applying $\mathfrak{L}_{m, p}$ to those of $F_{n-1, m}$. Finally, define $\vec{\alpha}:=\left(a_{0}, \ldots, a_{p}, b_{0}, \ldots, b_{p-2}\right)$.

The main result of [10] is that the Landen transformation satisfies

$$
\phi_{m, p}^{n}(\vec{\alpha}) \rightarrow \frac{I}{\pi}
$$

as $n \rightarrow \infty$ if $I<\infty$. Furthermore, the convergence is of order $m$, that is,

$$
\left|\phi_{m, p}^{n+1}(\vec{\alpha})-\frac{I}{\pi}\right| \leq C\left|\phi_{m, p}^{n}(\vec{\alpha})-\frac{I}{\pi}\right|^{m} .
$$

This convergence result appears in $[\underline{6}$ for the case $m=2$ and the general case can be established along the same lines. See 8$]$ for details.

Adapting the rational Landen transformations into a numerical method for calculating $I$ involves a process with two parts. The first one is a symbolic calculation of the explicit algebraic formulae for the rational Landen transformation. The steps in this calculation are described in detail in Section 4 . The second one is the iteration of these formulas.

\section{Algorithm 1}

Input:

1) An integer $p$ : the degree of the denominator $A$.

2) An integer $m \geq 2$ : the order of the transformation.

\section{Output:}


The explicit formula for the transformation $\mathfrak{L}_{m, p}: \mathcal{R}^{2 p} \rightarrow \mathcal{R}^{2 p}$, as polynomials in the $a_{i}$ and $b_{j}$.

Note 2.1. Observe that, given $m$ and $p$, the map $\mathfrak{L}_{m, p}$ can be precomputed and the result can be stored for its use in the second algorithm. Therefore, the first algorithm carries a one-time cost and is not figured into the time of the method. This precomputation will be assumed in the discussion of the second algorithm.

\section{Algorithm 2}

\section{Input:}

1) A vector $\vec{\alpha}$ representing the coefficients of the rational integrand $F$.

2) An integer $m \geq 2$ : the order of convergence.

3) An integer $n \in \mathbb{N}$ : the number of iterations of the Landen map.

Output: The expression $\phi_{m, p}^{n}(\vec{\alpha})$ that approximates $I / \pi$.

\section{The evaluation of the polynomials $P_{m}$ and $Q_{m}$}

The algorithm described in Section 4 employs the polynomials

$$
P_{m}(x):=\sum_{j=0}^{\lfloor m / 2\rfloor}(-1)^{j}\left(\begin{array}{l}
m \\
2 j
\end{array}\right) x^{m-2 j}
$$

and

$$
Q_{m}(x):=\sum_{j=0}^{\lfloor(m-1) / 2\rfloor}(-1)^{j}\left(\begin{array}{c}
m \\
2 j+1
\end{array}\right) x^{m-(2 j+1)} .
$$

The integration algorithm discussed here is based on the fact that the rational function

$$
R_{m}(x):=\frac{P_{m}(x)}{Q_{m}(x)}
$$

satisfies

$$
\cot (m \theta)=R_{m}(\cot \theta) .
$$

For instance, for $m=2$, we have

$$
P_{2}(x)=x^{2}-1, \text { and } Q_{2}(x)=2 x .
$$

Note 3.1. This trigonometric property is instrumental in the proof that the integral of $F$ is the same as that of $F_{1}$. See [10] for details.

\section{The ALGORIThM}

In this section we describe each of the steps in the first algorithm. This algorithm has been implemented in Mathematica 6.0.

Step 1. Construct the polynomial

$$
H(x)=\sum_{i=0}^{p} h_{i} x^{p-i}
$$

defined by

$$
H(x):=\operatorname{Res}_{z}\left(A(z), P_{m}(z)-x Q_{m}(z)\right),
$$


where $\operatorname{Res}_{z}$ denotes the resultant in the variable $z$. The degrees of the polynomials involved in (4.2) are $p=\operatorname{deg} A$ and $m=\operatorname{deg}\left(P_{m}(z)-x Q_{m}(z)\right)$, respectively.

The resultant. Given two polynomials $\alpha(t)$ and $\beta(t)$, the resultant of $\alpha$ and $\beta$ is defined by

$$
\operatorname{Res}(\alpha, \beta):=\prod_{i=1}^{r} \prod_{j=1}^{s}\left(y_{j}-x_{i}\right),
$$

where $x_{i}$ are the roots of $\alpha(t)=0$ and $y_{j}$ are the roots of $\beta(t)=0$.

The resultant of two polynomials can be computed as the determinant of the Sylvester matrix formed by their coefficients; see [7]. For instance, if

$$
\alpha(t)=a_{0}+a_{1} t+a_{2} t^{2}+a_{3} t^{3} \text { and } \beta(t)=b_{0}+b_{1} t+b_{2} t^{2},
$$

then the Sylvester matrix is defined by

$$
S_{3,2}:=\left(\begin{array}{cccccc}
a_{3} & a_{2} & a_{1} & a_{0} & 0 & 0 \\
0 & a_{3} & a_{2} & a_{1} & a_{0} & 0 \\
0 & 0 & a_{3} & a_{2} & a_{1} & a_{0} \\
b_{2} & b_{1} & b_{0} & 0 & 0 & 0 \\
0 & b_{2} & b_{1} & b_{0} & 0 & 0 \\
0 & 0 & b_{2} & b_{1} & b_{0} & 0 \\
0 & 0 & 0 & b_{2} & b_{1} & b_{0}
\end{array}\right)
$$

and it is a square matrix of size $\operatorname{deg}(\alpha)+\operatorname{deg}(\beta)+2=7$. The resultant of $\alpha(t)$ and $\beta(t)$ in (4.4) is

$$
\begin{aligned}
\operatorname{Res}(\alpha, \beta)= & a_{3}^{2} b_{0}^{3}-a_{2} a_{3} b_{0}^{2} b_{1}+a_{1} a_{3} b_{0} b_{1}^{2}-a_{0} a_{3} b_{1}^{3}+a_{2}^{2} b_{0}^{2} b_{2} \\
& -2 a_{1} a_{3} b_{0}^{2} b_{2}-a_{1} a_{2} b_{0} b_{1} q_{2}+3 a_{0} a_{3} b_{0} b_{1} b_{2}+a_{0} a_{2} b_{1}^{2} b_{2} \\
& +a_{1}^{2} b_{0} b_{2}^{2}-2 a_{0} a_{2} b_{0} b_{2}^{2}-a_{0} a_{1} b_{1} b_{2}^{2}+a_{0}^{2} b_{2}^{3} .
\end{aligned}
$$

In general, the resultant of two polynomials is a polynomial in their coefficients.

Note 4.1. The polynomial $H$ has the same degree as $A$, the denominator of the integrand $R(x)$. It will become the denominator of the new rational function. Its coefficients $h_{i}$ are polynomials in those of $A$. The calculation of $H$ can be obtained by evaluating the determinant of a square matrix of dimension $\operatorname{deg}(A)+\operatorname{deg}\left(P_{m}\right)+$ $2=p+m+2$. For instance, for $m=2$ and

$$
A(x)=a_{0} x^{4}+a_{1} x^{3}+a_{2} x^{2}+a_{1} x+a_{4},
$$

we obtain

$$
\begin{aligned}
H(x) & =16 a_{0} a_{4} x^{4}+8\left(a_{1} a_{4}-a_{0} a_{3}\right) x^{3}+4\left(a_{0} a_{2}-a_{1} a_{3}+4 a_{0} a_{4}+a_{2} a_{4}\right) x^{2} \\
& +2\left(-a_{0} a_{1}+a_{1} a_{2}-3 a_{0} a_{3}-a_{2} a_{3}+3 a_{1} a_{4}+a_{3} a_{4}\right) x \\
& +\left(a_{0}-a_{1}+a_{2}-a_{3}+a_{4}\right)\left(a_{0}+a_{1}+a_{2}+a_{3}+a_{4}\right) .
\end{aligned}
$$

Step 2. Form the polynomial

$$
E(x)=\sum_{i=0}^{p} h_{i}\left(P_{m}(x)\right)^{p-i}\left(Q_{m}(x)\right)^{i} .
$$


Step 3. The polynomial $E(x)$ formed in Step 2 is a multiple of the denominator $A(x)$. Compute the quotient

$$
Z(x)=\frac{E(x)}{A(x)}
$$

and write it as

$$
Z(x)=\sum_{k=0}^{r} z_{k} x^{r-k}, \text { with } r=p(m-1) .
$$

Step 4. Compute the product

$$
C(x)=B(x) Z(x)
$$

and write it as

$$
C(x)=\sum_{k=0}^{s} c_{k} x^{s-k}, \text { with } s=m p-2 .
$$

Step 5. Form the expression

$$
T_{x}(a, b):=\sum_{j=0}^{x}(-1)^{a-x+j}\left(\begin{array}{c}
a \\
x-j
\end{array}\right)\left(\begin{array}{l}
b \\
j
\end{array}\right)
$$

for $a, b, x \in \mathbb{N}$.

Step 6. Define the expressions

$$
\begin{aligned}
M_{1}(j, \alpha, \beta, \gamma, m, p) & :=(-1)^{j+\alpha-\beta} c_{2 j} \frac{2^{2(\alpha-\beta)} \alpha}{2 \alpha-\beta}\left(\begin{array}{c}
2 \alpha-\beta \\
\beta
\end{array}\right)\left(\begin{array}{c}
\nu-\alpha-1+\beta \\
\gamma
\end{array}\right) \times \\
& \times\left[T_{\lambda+\alpha m}(2 j, s-2 j)+T_{\lambda-\alpha m}(2 j, s-2 j)\right]
\end{aligned}
$$

and

$$
\begin{aligned}
M_{2}(j, \alpha, \beta, \gamma, m, p) & :=(-1)^{j+\beta} c_{2 j+1} 2^{2 \beta+1}\left(\begin{array}{c}
\alpha+\beta \\
2 \beta+1
\end{array}\right)\left(\begin{array}{c}
\nu-2-\beta \\
\gamma
\end{array}\right) \times \\
& \times\left[T_{\lambda+\alpha m}(2 j+1, s-2 j-1)-T_{\lambda-\alpha m}(2 j+1, s-2 j-1)\right],
\end{aligned}
$$

with $\nu:=p / 2$ and $\lambda:=(m p-2) / 2$. 
Step 7. Define

$$
\begin{aligned}
J(x) & :=\frac{1}{2^{s}} \sum_{\gamma=0}^{\nu-1}\left(\left(\begin{array}{c}
\nu-1 \\
\gamma
\end{array}\right) \sum_{j=0}^{\lambda}(-1)^{j} c_{2 j} T_{\lambda}(2 j, s-2 j)\right) x^{2 \gamma}+ \\
& +\frac{1}{2^{s}} \sum_{\gamma=0}^{\nu-2}\left(\sum_{j=0}^{\lambda} \sum_{\alpha=1}^{\nu-1-\gamma} \sum_{\beta=0}^{\alpha} M_{1}(j, \alpha, \beta, \gamma, m, p)\right) x^{2 \gamma} \\
& +\frac{1}{2^{s}} \sum_{\gamma=1}^{\nu-1}\left(\sum_{j=0}^{\lambda} \sum_{\alpha=\nu-\gamma}^{\nu-1} \sum_{\beta=\alpha-\nu+\gamma+1}^{\alpha} M_{1}(j, \alpha, \beta, \gamma, m, p)\right) x^{2 \gamma} \\
& +\frac{1}{2^{s}} \sum_{\gamma=0}^{\nu-2}\left(\sum_{j=0}^{\lambda-1} \sum_{\alpha=1}^{\nu-1-\gamma} \sum_{\beta=0}^{\alpha-1} M_{2}(j, \alpha, \beta, \gamma, m, p)\right) x^{2 \gamma+1} \\
& +\frac{1}{2^{s}} \sum_{\gamma=1}^{\nu-2}\left(\sum_{j=0}^{\lambda-1} \sum_{\alpha=\nu-\gamma}^{\nu-1} \sum_{\beta=0}^{\alpha-1} M_{2}(j, \alpha, \beta, \gamma, m, p)\right) x^{2 \gamma+1} .
\end{aligned}
$$

Step 8. The new rational function is defined by

$$
F_{1, m}(x):=\frac{J(x)}{H(x)} .
$$

It satisfies (2.4).

The reader is referred to [10] for the proofs of the formulas describing this algorithm.

\section{EXAMPLES}

In this section we give examples that illustrate the rational Landen transformations.

Example 5.1. We provide a step by step construction of the rational Landen transformation of order $m=2$ for the function

$$
F(x)=\frac{b_{0} x^{4}+b_{1} x^{3}+b_{2} x^{2}+b_{3} x+b_{4}}{a_{0} x^{6}+a_{1} x^{5}+a_{2} x^{4}+a_{3} x^{3}+a_{4} x^{2}+a_{5} x+a_{6}} .
$$

The goal is to produce a function $F_{1,6}(x)$ with the same integral as $F(x)$.

Note 5.1. The special case $b_{1}=b_{3}=a_{1}=a_{3}=a_{5}=0$ was the first example of this new type of transformation. It appears in [1].

Note 5.2. The choice of $m=2$ requires the evaluation of the polynomials $P_{2}(x)=$ $x^{2}-1$ and $Q_{2}(x)=2 x$. These are computed directly from (3.1) and (3.2). 
Step 1 computes the polynomial $H(x)$ from (4.2). The Mathematica command Resultant yields the expressions

$$
\begin{aligned}
h_{0}= & 64 a_{0} a_{6} \\
h_{1}= & -32\left(a_{0} a_{5}-a_{1} a_{6}\right) \\
h_{2}= & 16\left(a_{0} a_{4}-a_{1} a_{5}+6 a_{0} a_{6}+a_{2} a_{6}\right) \\
h_{3}= & -8\left(a_{0} a_{3}-a_{1} a_{4}+5 a_{0} a_{5}+a_{2} a_{5}-5 a_{1} a_{6}-a_{3} a_{6}\right) \\
h_{4}= & 4\left(a_{0} a_{2}-a_{1} a_{3}+4 a_{0} a_{4}+a_{2} a_{4}-4 a_{1} a_{5}-a_{3} a_{5}+9 a_{0} a_{6}+4 a_{2} a_{6}+a_{4} a_{6}\right) \\
h_{5}= & -2\left(a_{0} a_{1}-a_{1} a_{2}+3 a_{0} a_{3}+a_{2} a_{3}-3 a_{1} a_{4}-a_{3} a_{4}+5 a_{0} a_{5}\right) \\
& -2\left(3 a_{2} a_{5}+a_{4} a_{5}-5 a_{1} a_{6}-3 a_{3} a_{6}-a_{5} a_{6}\right) \\
h_{6}= & \left(a_{0}-a_{1}+a_{2}-a_{3}+a_{4}-a_{5}+a_{6}\right)\left(a_{0}+a_{1}+a_{2}+a_{3}+a_{4}+a_{5}+a_{6}\right) .
\end{aligned}
$$

The polynomial $H(x)$ is the denominator of the new rational function obtained as a product of the Landen transformation.

Step 2 computes the polynomial $E(x)$ from (4.6). In this example, this is a polynomial of degree $12(=m p)$, that we write as

$$
E(x)=\sum_{i=0}^{12} e_{i} x^{12-i}
$$

The symbolic expansion of (4.6) produces

$$
\begin{aligned}
& e_{0}=e_{12}=64 a_{0} a_{6} \\
& e_{1}=-e_{11}=64\left(a_{0} a_{5}-a_{1} a_{6}\right) \\
& e_{2}=e_{10}=64\left(a_{0} a_{4}-a_{1} a_{5}+a_{2} a_{6}\right) \\
& e_{3}=-e_{9}=64\left(a_{0} a_{3}-a_{1} a_{4}+a_{2} a_{5}-a_{3} a_{6}\right) \\
& e_{4}=e_{8}=64\left(a_{0} a_{2}-a_{1} a_{3}+a_{2} a_{4}-a_{3} a_{5}+a_{4} a_{6}\right) \\
& e_{5}=-e_{7}=64\left(a_{0} a_{1}-a_{1} a_{2}+a_{2} a_{3}-a_{3} a_{4}+a_{4} a_{5}-a_{5} a_{6}\right) \\
& e_{6}=64\left(a_{0}^{2}-a_{1}^{2}+a_{2}^{2}-a_{3}^{2}+a_{4}^{2}-a_{5}^{2}+a_{6}^{2}\right) .
\end{aligned}
$$

Step 3 computes the quotient of $E(x)$, produced in Step 2, and $A(x)$, the denominator of the original integrand. In the example discussed now we obtain

$$
Z(x)=64\left(a_{6} x^{6}-a_{5} x^{5}+a_{4} x^{4}-a_{3} x^{3}+a_{2} x^{2}-a_{1} x+a_{0}\right) .
$$

Step 4 simply evaluates the product $C(x)=B(x) Z(x)$, where $B(x)$ is the numerator of the original integrand and $Z(x)$ comes from Step 3. The polynomial $C(x)$ is written as

$$
C(x)=\sum_{k=0}^{10} c_{k} x^{10-k},
$$


with

$$
\begin{aligned}
c_{0} & =64 a_{6} b_{0} \\
c_{1} & =-64\left(a_{5} b_{0}-a_{6} b_{1}\right) \\
c_{2} & =64\left(a_{4} b_{0}-a_{5} b_{1}+a_{6} b_{2}\right) \\
c_{3} & =-64\left(a_{3} b_{0}-a_{4} b_{1}+a_{5} b_{2}-a_{6} b_{3}\right) \\
c_{4} & =64\left(a_{2} b_{0}-a_{3} b_{1}+a_{4} b_{2}-a_{5} b_{3}+a_{6} b_{4}\right) \\
c_{5} & =-64\left(a_{1} b_{0}-a_{2} b_{1}+a_{3} b_{2}-a_{4} b_{3}+a_{5} b_{4}\right) \\
c_{6} & =64\left(a_{0} b_{0}-a_{1} b_{1}+a_{2} b_{2}-a_{3} b_{3}+a_{4} b_{4}\right) \\
c_{7} & =64\left(a_{0} b_{1}-a_{1} b_{2}+a_{2} b_{3}-a_{3} b_{4}\right) \\
c_{8} & =64\left(a_{0} b_{2}-a_{1} b_{3}+a_{2} b_{4}\right) \\
c_{9} & =64\left(a_{0} b_{3}-a_{1} b_{4}\right) \\
c_{10} & =64 a_{0} b_{4} .
\end{aligned}
$$

Step 7 combines the functions defined in Step 5 and 6 to produce the new numerator

$$
J(x)=\sum_{k=0}^{4} d_{k} x^{4-k},
$$

with

$$
\begin{aligned}
d_{0}= & 32\left(a_{6} b_{0}+a_{0} b_{4}\right) \\
d_{1}= & -16\left(a_{5} b_{0}-a_{6} b_{1}+a_{0} b_{3}-a_{1} b_{4}\right) \\
d_{2}= & 8\left(a_{4} b_{0}+3 a_{6} b_{0}-a_{5} b_{1}+a_{0} b_{2}+a_{6} b_{2}-a_{1} b_{3}+3 a_{0} b_{4}+a_{2} b_{4}\right) \\
d_{3}= & -4\left(a_{3} b_{0}+2 a_{5} b_{0}+a_{0} b_{1}-a_{4} b_{1}-2 a_{6} b_{1}-a_{1} b_{2}+a_{5} b_{2}\right)- \\
& -4\left(2 a_{0} b_{3}+a_{2} b_{3}-a_{6} b_{3}-2 a_{1} b_{4}-a_{3} b_{4}\right) \\
d_{4}= & 2\left(a_{0} b_{0}+a_{2} b_{0}+a_{4} b_{0}+a_{6} b_{0}-a_{1} b_{1}-a_{3} b_{1}-a_{5} b_{1}\right)+ \\
& +2\left(a_{0} b_{2}+a_{2} b_{2}+a_{4} b_{2}+a_{6} b_{2}-a_{1} b_{3}-a_{3} b_{3}+a_{0} b_{4}+a_{2} b_{4}+a_{4} b_{4}+a_{6} b_{4}\right) .
\end{aligned}
$$

Note 5.3. Given a rational function of order $p$ and a choice of method of order $m$, the calculation of $H$ and $J$ illustrated here is done once. We have produced a transformation sending

$$
F(x)=\frac{b_{0} x^{4}+b_{1} x^{3}+b_{2} x^{2}+b_{3} x+b_{4}}{a_{0} x^{6}+a_{1} x^{5}+a_{2} x^{4}+a_{3} x^{3}+a_{4} x^{2}+a_{5} x+a_{6}}
$$

to

$$
F_{1,6}(x):=\mathfrak{L}_{6,2} F(x)=\frac{d_{0} x^{4}+d_{1} x^{3}+d_{2} x^{2}+d_{3} x+d_{4}}{h_{0} x^{6}+h_{1} x^{5}+h_{2} x^{4}+h_{3} x^{3}+h_{4} x^{2}+h_{5} x+h_{6}}
$$

with the new coefficients given as above. We expect to produce a precomputed array of formulas, indexed by $(p, m)$, to be made available to the community.

In the next series of examples, we will assume that the formulas for the Landen transformations have been precomputed. 
Example 5.2. The rational function

$$
F_{0}(x)=\frac{1}{x^{2}+4 x+15}
$$

satisfies

$$
I:=\int_{-\infty}^{\infty} F_{0}(x) d x=\frac{\pi}{\sqrt{11}}
$$

with numerical value

$$
I \sim 0.94722582509948293643
$$

We now employ the algorithm, with a method of order 2, to obtain the sequence of rational functions

$$
F_{n, 2}(x)=\frac{c_{n}}{x^{2}+a_{n} x+b_{n}},
$$

with $F_{0,2}(x)=F_{0}(x)$, and the property that

$$
I=\int_{-\infty}^{\infty} F_{n, 2}(x) d x=c_{n} \int_{-\infty}^{\infty} \frac{d x}{x^{2}+a_{n} x+b_{n}} .
$$

The convergence analysis described in [10] shows that $a_{n} \rightarrow 0, b_{n} \rightarrow 1$, thus

$$
I=\lim _{n \rightarrow \infty} \pi c_{n} .
$$

Even though the limiting value of the integral depends only upon the terms $c_{n}$, the formulas to generate these values also involve $a_{n}$ and $b_{n}$. Therefore one must store the current value of all the parameters. The first few of them are shown in the next table:

\begin{tabular}{|c|c|c|c|}
\hline \hline$n$ & $c_{n}$ & $a_{n}$ & $b_{n}$ \\
\hline \hline 0 & 1 & 4 & 15 \\
\hline 1 & $\frac{8}{15}$ & $\frac{28}{15}$ & 4 \\
\hline 2 & $\frac{1}{3}$ & $\frac{7}{10}$ & $\frac{4841}{3600}$ \\
\hline 3 & $\frac{8441}{29046}$ & $\frac{8687}{96820}$ & $\frac{64900081}{69710400}$ \\
\hline
\end{tabular}

TABLE 1. Rational Landen of order 2

Note 5.4. The expression $\pi c_{n}$ gives an approximation to the integral of $F_{0}(x)$. For example, for $n=6$, we find that

$$
c_{6}=\frac{3471070386673821384824326347489289738211683509253931254760471}{11512238093504492278949475398059063785494372327433955614454608}
$$


and $\pi c_{6}$ agrees with the integral of $F_{0}$ up to 13 digits. The coefficients $\left(a_{n}, b_{n}, c_{n}\right)$ are rational numbers and their height increases with $n$. Recall that the height of a rational number, written in irreducible form as $x=\frac{u}{v}$, is

$$
h(x)=\operatorname{Max}\{|u|,|v|\} .
$$

For example, at $n=10$, the heights of $a_{10}, b_{10}, c_{10}$ have approximately 1000 digits. At this stage, the value $\pi c_{10}$ gives 196 digits of the integral. Naturally, this increases the complexity of the calculations if we use exact arithmetic. An interesting way out of this problem is to replace the rational number $c_{n}$ by the truncation of its continued fraction. For example, the first 20 terms of the continued fraction of $c_{10}$, a rational number of height only 6 , differs from $c_{10}$ by less than $10^{-24}$. Details will be given in a future publication.

Example 5.3. Mathematica 6.0 shows that if

$$
F(x)=\frac{1}{x^{4}+6 x^{3}+16 x^{2}+21 x+13}
$$

then

$$
I:=\int_{-\infty}^{\infty} F(x) d x=2 \pi \sqrt{\frac{2}{111}(\sqrt{37}-5) .}
$$

We employ the identity (1.3) to map the problem to the interval $[0,1]$. The new rational function

$$
\begin{aligned}
g(x) & :=\frac{60 x^{6}-288 x^{5}+584 x^{4}-648 x^{3}+422 x^{2}-156 x+26}{\left(3 x^{4}-15 x^{3}+31 x^{2}-31 x+13\right)\left(57 x^{4}-153 x^{3}+157 x^{2}-73 x+13\right)} \\
& =\frac{60 x^{6}-288 x^{5}+584 x^{4}-648 x^{3}+422 x^{2}-156 x+26}{171 x^{8}-1314 x^{7}+4533 x^{6}-9084 x^{5}+11485 x^{4}-9314 x^{3}+4707 x^{2}-1352 x+169}
\end{aligned}
$$

satisfies

$$
\int_{0}^{1} g(x) d x=\int_{-\infty}^{\infty} F(x) d x
$$

We now compare the numerical approximation to the integral of $g$ over $[0,1]$ computed with the methods described here, with a numerical integration using the trapezoidal rule. A more systematic comparison with more sophisticated classical numerical schemes will be described elsewhere.

The trapezoidal rule states that

$$
\int_{a}^{b} g(x) d x=\frac{h}{2}(g(a)+g(b))-\frac{1}{12} h^{3} g^{\prime \prime}(\xi),
$$

where $h=b-a$ and $\xi \in[a, b]$. Define

$$
M:=\operatorname{Max}\left\{\left|g^{\prime \prime}(t)\right|: a \leq t \leq b\right\} .
$$

Then the error term, namely the second term in (5.18) is bounded by $M h^{3} / 12$. To obtain an approximation to the integral $I$ in (5.16), we choose $n \in \mathbb{N}$, partition $[0,1]$ into $n$ intervals of equal length $h=1 / n$ and apply the trapezoidal rule to each subinterval. This yields the expression

$$
I_{n}:=\int_{0}^{1} g(x) d x \sim \frac{1}{2 n}\left(g_{0}+\sum_{i=1}^{n-2} g_{i}+g_{n}\right)-\frac{1}{12 n^{3}} \sum_{i=1}^{n-1} g^{\prime \prime}\left(\xi_{i}\right)
$$


where $g_{i}=g(i / n)$ and $\frac{i-1}{n} \leq \xi_{i} \leq \frac{i}{n}$. The total error in this approximation is bounded by $M / 12 n^{2}$. To compute the approximation $I_{n}$ to $I$ requieres the $n$ values $\left\{g_{i}: 0 \leq i \leq n\right\}$. The relative error $\left(I-I_{n}\right) / I$ for $n=100$ is $5.29805 \times 10^{-6}$. It drops to $3.1505 \times 10^{-8}$ for $n=1000$ and to $2.9445 \times 10^{-10}$ for $n=10000$.

We now use the method of rational Landen transformations of order $m$ to produce approximations to the integral $I$ of $F(x)$ over $\mathbb{R}$. Recall that the method yields a family of rational functions $R_{n, m}(x)$ with integral $I$. For example, the first two functions for a method of order 2 are

$$
\begin{aligned}
R_{1,2}(x) & =\frac{4\left(2 x^{2}+6 x+15\right)}{208 x^{4}+456 x^{3}+600 x^{2}+396 x+171} \\
R_{2,2}(x) & =\frac{8\left(13848 x^{2}+11652 x+11531\right)}{569088 x^{4}-35136 x^{3}+756384 x^{2}-8616 x+232537} .
\end{aligned}
$$

The approximations to $I$ are then obtained from

$$
\operatorname{app}_{n, m}:=\frac{\text { Constant term in the numerator of } R_{n, m}(x)}{\text { Constant term in the denominator of } R_{n, m}(x)} \times \pi .
$$

The next table shows the relative errors

$$
\operatorname{rel}_{n, m}:=\frac{\left|\operatorname{app}_{n, m}-I\right|}{|I|}
$$

for $2 \leq m \leq 6$ and $2 \leq n \leq 5$.

\begin{tabular}{|c|c|c|c|c|c|}
\hline \hline$n$ & $m=2$ & $m=3$ & $m=4$ & $m=5$ & $m=6$ \\
\hline \hline 2 & 0.30314 & 0.022076 & 0.0021170 & $2.2646 \times 10^{-6}$ & $6.3257 \times 10^{-7}$ \\
\hline 3 & 0.058475 & 0.000035272 & $5.2932 \times 10^{-12}$ & $2.9440 \times 10^{-23}$ & $4.4813 \times 10^{-40}$ \\
\hline 4 & 0.0021170 & $3.2713 \times 10^{-15}$ & $2.0616 \times 10^{-47}$ & $1.9758 \times 10^{-115}$ & $3.6655 \times 10^{-239}$ \\
\hline 5 & $3.2700 \times 10^{-6}$ & $3.6952 \times 10^{-45}$ & $5.3750 \times 10^{-190}$ & $3.1671 \times 10^{-577}$ & $4.0442 \times 10^{-1434}$ \\
\hline \hline
\end{tabular}

TABLE 2. Relative error for the numerical evaluation of $I_{n}$.

This table contains clear evidence to support the convergence orders claimed in (2.8).

Example 5.4. The rational Landen transformations can be used to evaluate

$$
\int_{-\infty}^{\infty} \frac{d x}{(x-2)^{2}+\epsilon^{2}}=\frac{\pi}{\epsilon}
$$

for $\epsilon>0$ small. This example illustrates the fact that the proposed method converges, even when the integrand has poles very close to the real axis. A systematic 
description of the sensitivity of the iteration with respect to the parameter $\epsilon$, will be presented elsewhere.

For fixed $\epsilon>0$, we apply a method of order 2 to (5.23). This produces a sequence of rational functions of the form

$$
R_{n}(x)=\frac{b_{0, n}}{a_{0, n}+a_{1, n} x+a_{2, n} x^{2}}
$$

that satisfy

$$
\int_{-\infty}^{\infty} R_{n}(x) d x=\frac{\pi}{\epsilon}
$$

The explicit Landen transformation of order 2 is given by

$$
\begin{aligned}
& a_{0, n+1}=\left(a_{0, n}-a_{1, n}+a_{2, n}\right)\left(a_{0, n}+a_{1, n}+a_{2, n}\right) \\
& a_{1, n+1}=2 a_{1, n}\left(a_{0, n}-a_{2, n}\right) \\
& a_{2, n+1}=4 a_{0, n} a_{2, n} \\
& b_{0, n+1}=2 b_{0, n}\left(a_{0, n}+a_{2, n}\right)
\end{aligned}
$$

with initial conditions

$$
a_{0,0}=4+\epsilon^{2}, a_{1,0}=-4, a_{2,0}=1, b_{0,0}=1 .
$$

The sequence $R_{n}(x)$ has coefficients that depend upon the parameter $\epsilon$. For example,

$$
R_{1}(x)=\frac{2\left(5+\epsilon^{2}\right)}{4\left(4+\epsilon^{2}\right) x^{2}-8\left(3+\epsilon^{2}\right) x+\left(1+\epsilon^{2}\right)\left(9+\epsilon^{2}\right)}
$$

and

$$
R_{2}(x)=\frac{4\left(5+\epsilon^{2}\right)\left(25+14 \epsilon^{2}+\epsilon^{4}\right)}{\left(1+6 \epsilon^{2}+\epsilon^{4}\right)\left(49+22 \epsilon^{2}+\epsilon^{4}\right)-16(-1+\epsilon)(1+\epsilon)\left(3+\epsilon^{2}\right)\left(7+\epsilon^{2}\right) x+16\left(1+\epsilon^{2}\right)\left(4+\epsilon^{2}\right)\left(9+\epsilon^{2}\right)} .
$$

The theory described above shows that, for fixed $\epsilon>0$ and $n \rightarrow \infty$, the sequences

$$
\frac{a_{0, n}}{b_{0, n}} \rightarrow L, \quad \frac{a_{1, n}}{b_{0, n}} \rightarrow 0, \quad \frac{a_{2, n}}{b_{0, n}} \rightarrow L
$$

converge to the stated limits. Moreover, the invariance of (5.23) under the transformations given in (5.26) show that $L=\epsilon$.

Define the error

$$
\operatorname{err}_{n}:=\left(\left(\frac{a_{0, n}}{b_{0, n}}-\epsilon\right)^{2}+\left(\frac{a_{1, n}}{b_{0, n}}\right)^{2}+\left(\frac{a_{2, n}}{b_{0, n}}-\epsilon\right)^{2}\right)^{1 / 2} .
$$

Then Table 3 shows the ratios err ${ }_{16} / \operatorname{err}_{15}$ obtained after 15 iterations of $(5.26)$ for methods of order 2 and 3 . The calculations are done with $10^{6}$ digit precision.

The data in Table 3 shows the exponential decay of the error. Given a tolerance $\delta>0$, we have observed that the number of steps required to achieve $\operatorname{err}_{n}<\delta$ increases as $\epsilon \rightarrow 0$. A quantitative description of this phenomena is in preparation and it will be reported elsewhere. 


\begin{tabular}{|c|c|c|}
\hline \hline$\epsilon$ & order 2 & order 3 \\
\hline \hline .1 & $3.58047 \times 10^{-569}$ & $3.49118 \times 10^{-497802}$ \\
\hline .01 & $1.36862 \times 10^{-57}$ & $4.24935 \times 10^{-49853}$ \\
\hline .001 & $2.07254 \times 10^{-6}$ & $4.73905 \times 10^{-4986}$ \\
\hline .0001 & $2.16805 \times 10^{-2}$ & $3.48094 \times 10^{-499}$ \\
\hline .00001 & $4.68150 \times 10^{-1}$ & $1.62880 \times 10^{-50}$ \\
\hline
\end{tabular}

TABLE 3. The quotient $\operatorname{err}_{16} / \operatorname{err}_{15}$ as a function of the parameter $\epsilon$.

\section{Conclusions}

We have described the rational Landen transformations and their use in the numerical integration of rational functions. We have exhibited fast convergence of this method and presented an example comparing it to the classical integration schemes.

A systematic comparative analysis of this method with respect to standard numerical algorithms will be discussed elsewhere. An interesting challenging problem is to extend the use of rational Landen transformations to produce fast numerical integrators for arbitrary functions. In particular the method is well suited for the numerical integration of meromorphic function with poles off the real line.

Acknowledgments. The first author is partially funded by the AARMS Director's Postdoctoral Fellowship. The work of the second author was partially funded by NSF-DMS 0070567. The authors wish to thank a referee for suggesting the integral described in the last example.

\section{REFERENCES}

[1] G. Boros and V. Moll. A rational Landen transformation. The case of degree 6. In Knopp G. Mendoza E.T. Quinto E. L. Grinberg S. Berhanu M, editor, Contemporay Mathematics. Analysis, Geometry, Number Theory: The Mathematics of Leon Ehrenpreis, volume 251, pages 83-89. American Mathematical Society, 2000.

[2] G. Boros and V. Moll. Landen transformation and the integration of rational functions. Math. Comp., 71:649-668, 2001.

[3] J. M. Borwein and P. B. Borwein. Pi and the AGM- A study in analytic number theory and computational complexity. Wiley, New York, 1st edition, 1987.

[4] P. J. Davis and P. Rabinowitz. Methods of Numerical Integration, volume 2nd. Academic Press, New York, 1984.

[5] K. F. Gauss. Arithmetisch Geometrisches Mittel. Werke, 3:361-432, 1799.

[6] J. Hubbard and V. Moll. A geometric view of rational Landen transformation. Bull. London Math. Soc., 35:293-301, 2003.

[7] S. Lang. Algebra. Springer-Verlag, revised third edition edition, 2002.

[8] D. Manna. Landen transformations. PhD thesis, Tulane University, 2005. 
[9] D. Manna and V. Moll. Landen Survey. MSRI Publications: Probabilty, Geometry and Integrable Systems. In honor of Henry McKean 75th birthday, 55:201-233, 2007.

[10] D. Manna and V. Moll. Rational Landen transformations on $\mathbb{R}$. Math. Comp., 76:2023-2043, 2007.

[11] C. W. Ueberhuber. Numerical Computation, volume 2. Springer Verlag, 1st edition, 1997.

Department of Mathematics and Statistics, Dalhousie University, Halifax, Nova ScoTia, CANADA B3H $3 \mathrm{~J} 5$

E-mail address: dmanna@mathstat.dal.ca

Department of Mathematics, Tulane University, New Orleans, LA 70118

E-mail address: vhm@math.tulane.edu 